\title{
Treatment of Stable Angina with a New Fixed-Dose Combination of Ivabradine and Metoprolol: Effectiveness and Tolerability in Routine Clinical Practice
}

Dimitar Divchev · Georg Stöckl · On behalf of the study investigators

Received: September 1, 2017 / Published online: November 7, 2017

(C) The Author(s) 2017. This article is an open access publication

\begin{abstract}
Introduction: In this prospective, multicenter, observational cohort study, the effectiveness and tolerability of the first fixed-dose combination (FDC) formulation of the selective heart rate reducing agent ivabradine and the betablocker metoprolol was evaluated in stable angina pectoris (AP) patients in a clinical practice setting.

Methods: Stable AP outpatients received a FDC of ivabradine and metoprolol (b.i.d.) for 4 months, in addition to cardiovascular standard therapy. Resting heart rate (HR), number of angina attacks, short-acting nitrate consumption, severity of symptoms (assessed by patient judgment and documented by CCS score) and tolerability were documented. Medication adherence was assessed by a modified four-item Morisky questionnaire. Descriptive statistics were performed on all data.
\end{abstract}

Enhanced content To view enhanced content for this article go to http://www.medengine.com/Redeem/ 76CCF0600FBD0931

D. Divchev $(\bowtie)$

Department of Cardiology and Angiology,

University Hospital Marburg, Baldingerstrasse,

35043 Marburg, Germany

e-mail: dimitar.divchev@med.uni-marburg.de

G. Stöckl

Department of Medical Affairs, Servier Deutschland

GmbH, Munich, Germany
Results: A total of 747 stable AP patients (mean age, 66.4 years, $62 \%$ male, $50 \%$ and $31 \%$ with previous PCI and myocardial infarction, respectively) were included. Apart from ivabradine and beta-blockers as free combination, most frequently used concomitant standard medications at baseline were aspirin (68\%), statins (71\%), ACEI/AT1-blockers (76\%), diuretics (35\%), and calcium antagonists (15\%). Highly prevalent comorbidities were hypertension (86\%), hyperlipidemia (65\%), and diabetes (35\%). After 4 months, switch to treatment with the FDC was associated with a significant reduction in mean HR by $10 \mathrm{bpm}$. Proportion of patients with $\geq 1$ angina attacks/week decreased from 38 to $7 \%$. Patients in CCS class 1 increased (25 to 63\%), while they decreased in CCS class 3 (19 to 5\%). Medication adherence was also significantly improved $(p<0.001$ for all changes from baseline). Mostly mild adverse events were documented in $5.4 \%$ of patients.

Conclusions: In these stable AP patients in a real-life setting, treatment with a FDC of ivabradine and metoprolol was associated with reduced HR and angina symptoms, while exercise capacity (CCS score) was improved. These effects may be mainly mediated by the increased medication adherence of patients observed with use of the FDC formulation.

Funding: Servier Trial registration number: ISRCTN51906157 
Keywords: Angina attacks; Beta-blocker; CCS class; Fixed-dose combination; Heart rate reduction; Ivabradine; Medication adherence; Stable angina pectoris; Symptom improvement

\section{INTRODUCTION}

Elevated heart rate can disturb the myocardial oxygen balance by simultaneously increasing myocardial oxygen demand and limiting myocardial perfusion due to the shortening of diastolic perfusion time. Both mechanisms can either induce or worsen myocardial ischemia and thereby trigger angina symptoms and reduce exercise capacity [1]. As a consequence, there is a strong emphasis in guidelines on sufficient heart rate reduction as an important strategy to prevent oxygen imbalance and improve angina symptoms in stable AP patients $[2,3]$. To control heart rate and reduce symptoms, beta-blockers are mostly recommended as a first-line strategy. However, in clinical practice, their use can be limited due to their broad mode of action and resulting side effects $[4,5]$. Moreover, registry data have shown many patients to remain symptomatic under betablocker therapy $[6,7]$, indicating the need for combining heart rate reducing agents. In contrast to beta-blockers, ivabradine, an inhibitor of the funny channel pacemaker current $\left(I_{\mathrm{f}}\right)$, selectively targets heart rate without reducing myocardial contractility or relaxation [8-10]. In recent studies the mechanisms of anti-ischemic and anti-anginal efficacy of ivabradine were further elucidated. In patients with chronic stable coronary artery disease (CAD), ivabradine was associated with an increase of myocardial diastolic perfusion time, an enhanced coronary flow reserve, improved endothelial function, and enhanced coronary collateral flow [11-15].

The anti-ischemic and anti-anginal efficacy of ivabradine had been proven in randomized clinical trials [16-20], supporting its guideline recommended use for symptomatic treatment in patients with stable AP either alone, if beta-blockers are contraindicated, or in combination with them if symptoms persist despite beta-blocker therapy. Combining a beta-blocker with ivabradine has been shown to further reduce the heart rate, resulting in less myocardial ischemia and angina symptoms along with a higher exercise capacity in a clinical trial [20]. Similar results were observed in three large observational studies with stable AP patients in daily routine practice [21-23]. In particular, a post hoc analysis of the ADDITIONS (prActical Daily efficacy anD safety of ivabradine In combinaTION with beta-blockerS) study as well as a pooled analysis of these three observational cohorts have proven beneficial effects for adding ivabradine to an existing therapy with the most commonly prescribed beta-blocker metoprolol in real-life populations of stable CAD patients [24, 25].

Although only scarce data are available on medication adherence for symptomatic therapies in stable CAD patients, studies on secondary prevention after a first cardiovascular event requiring a percutaneous coronary intervention (PCI) suggest a rapid decline in adherence to newly prescribed medications such as, for example, beta-blockers [26]. This phenomenon can be observed especially in patients with co-morbidities and complex therapy regimen [27]. To reduce the tablet burden and thereby increasing medication adherence in AP patients, a fixed-dose combination (FDC) of metoprolol and ivabradine was developed. However, data is still lacking on use of a FDC of these two drugs. The current study therefore aimed to evaluate the effectiveness and tolerability of the first FDC formulation of metoprolol and ivabradine in routine clinical practice, and to detect effects on medication adherence.

\section{METHODS}

Stable AP patients fulfilling official criteria for treatment with the new ivabradine/metoprolol FDC (Implicor ${ }^{\mathrm{TM}} 5 / 25,5 / 50,7.5 / 25,7.5 / 50$ ) according to the approved $\mathrm{EU}$ indication were eligible for inclusion in this non-interventional observational cohort study by cardiologists, general practitioners and internists in an outpatient setting. Three visits were planned, consisting of baseline examination (visit 1), a control visit after 4 weeks (visit 2), and the final examination after 4 months (visit 3). All data 
was systematically documented using a standardized case report form (CRF).

All procedures followed were in accordance with the ethical standards of the responsible committee on human experimentation (institutional and national) and with the Helsinki Declaration of 1964, as revised in 2013. Informed consent was obtained from all patients for being included in the study. Ethical approval was granted by the independent ethics commission in Freiburg/Germany (FEKI). This trial is registered at controlled-trials.com with registration number ISRCTN51906157. Demographic data and general/disease-specific medical history, information about concomitant diseases, other relevant medical therapies and reasons for initiation of FDC treatment were recorded at baseline visit. According to the approved label, the starting dose of the FDC should be equivalent to the dosing of the individual components (ivabradine and metoprolol) before switch. If necessary to achieve symptom control, the dose could be adjusted during the study to a maximal dose of $7.5 \mathrm{mg}$ ivabradine and $50 \mathrm{mg}$ metoprolol twice daily. Dose reduction was possible in case of pronounced heart rate reduction $<50$ beats per minute (bpm) or occurrence of bradycardic symptoms during treatment.

Patient status was clinically documented at each visit by recording HR, number of weekly angina symptoms, use of short-acting nitrates, and CCS class. Other parameters assessed at baseline and/or during the course of the study included history of myocardial infarction (MI), revascularization therapies like percutaneous coronary intervention (PCI) or coronary artery bypass grafting (CABG), blood pressure profile, conduction disorders, use of pacemaker devices, NYHA class, left ventricular dysfunction (LVD). At visit 3, relevant changes in concomitant medications were analyzed. For assessment of the overall response rate to FDC treatment, response was defined as achieving a $\mathrm{HR}<70 \mathrm{bpm}$ and/or an absolute HR reduction of $\geq 10 \mathrm{bpm}$ at study end (visit 3). Furthermore, an evaluation of the overall effectiveness and tolerability of therapy was made using a physician's assessment scale with categories "very good", "good", "moderate", and "poor". Patient impression and satisfaction with the switch to a FDC treatment was documented by use of three open questions at visit 3 .

An important objective of this study was to evaluate possible effects of a switch to FDC therapy on medication adherence of patients (by reduction of pill burden). For this purpose, a modified four-item Morisky questionnaire [28] was used to assess medication adherence at baseline compared to study end (visit 3) after 4 months of treatment. Proportion of patients with "complete adherence", which was defined as the absence of any adherence-related problems reported in the questionnaire, was analyzed for both time points.

A number of additional subgroups were specified according to gender, age $(<1$ $\geq 75$ years), resting HR $(</ \geq 75 \mathrm{bpm})$, CCS class (I-IV), patients with or without PCI, LVD, and pre-treatment with ivabradine and/or metoprolol at baseline. All adverse events (AE) and adverse drug reactions (ADR) occurring during the study period had to be documented and assessed by the treating physicians on a standardized AE/ADR reporting form at each patient visit after baseline examination. Coding of all AE/ADR data was performed in accordance with the Medical Dictionary for Regulatory Activities (MedDRA) version 19.1.

Due to the observational design of the study, statistical analysis of the results was performed by a descriptive approach. Data are presented as mean values \pm standard deviations (SD) for continuous variables and numbers of patients and/or percentages for categorical variables. Analysis of effectiveness data was performed with data imputation according to the last value carried forward method (LVCF). Wilcoxon's signed-rank test, Fisher's exact test and Chi-square test were applied as appropriate for assessment of changes between baseline and follow-up visits. Corresponding $p$ values should be interpreted in a descriptive-exploratory way. The $p$ values reported are two-tailed and an alpha level of 0.05 was used to assess statistical significance. No correction of alpha level for multiple testing was performed. All study data were evaluated by an independent statistical institute (ANFOMED GmbH, Möhrendorf, Germany). All statistical analyses have been 
performed by means of the SAS software system (version 9.4 for Microsoft Windows 10; SAS Institute Inc., Cary, NC, USA).

\section{RESULTS}

\section{Baseline Characteristics}

A total of 747 patients with chronic stable AP (intention to treat population) were enrolled in this non-interventional study in 260 centers in Germany. Data of 741 patients (99.2\%) were available for all three visits. The mean study duration was 3.9 months (range, 0.107.9 months). The mean age of the cohort was $66.4 \pm 10.8$ years $(24.7 \% \geq 75$ years $), 62.4 \%$ of the patients were male. On average, patients suffered from CAD for 70.0 months and from AP for 59.4 months. $31.3 \%$ of the study cohort had a history of myocardial infarction (MI), 50.3 and $10.2 \%$ underwent PCI or CABG, respectively. $32.8 \%$ of the study cohort had left ventricular dysfunction (LVD), mean LV ejection fraction (LVEF) was $44.6 \pm 11.5 \%$. Valvular heart defects were present in $20.1 \%$, and conduction disturbances in $22.7 \%$ of patients. Nearly all participants presented with cardiovascular risk factors or concomitant diseases (Table 1), most commonly with hypertension (86.4\%), hyperlipidemia (65.2\%), obesity (42.0\%), type 2 diabetes (34.9\%), nicotine abuse (30.9\%), COPD (15.1\%), depression (13.8\%), and renal impairment (8.8\%).

Apart from ivabradine (mean daily dose $9.9 \mathrm{mg}$ ) and beta-blockers (metoprolol in $94.1 \%$ with a mean daily dose of $93.6 \mathrm{mg}$ ) as free combination, concomitant cardiovascular standard medication included statins (70.8\%), aspirin (67.5\%), ACE-inhibitors/AT1-blockers $(76.3 \%)$, diuretics $(34.5 \%)$, oral anticoagulants $(10.7 \%)$, calcium antagonists (15.4\%), aldosterone antagonists (8.7\%), and long-acting nitrates $(6.3 \%)$.

The main reasons of physicians for prescribing the FDC of metoprolol and ivabradine were to improve adherence by reducing tablet burden (89.2\%), and save therapy costs (42.0\%). Most frequently used metoprolol/ivabradine FDC dosage forms at the start of therapy were
Table 1 Baseline characteristics of study population

\begin{tabular}{|c|c|}
\hline Characteristics & $(N=747)$ \\
\hline \multicolumn{2}{|l|}{ Demographic characteristics } \\
\hline Age (years) & $66 \pm 10.8$ \\
\hline$\geq 75$ years & $184(25 \%)$ \\
\hline Sex (male) & $455(62 \%)$ \\
\hline Body mass index $\left(\mathrm{kg} / \mathrm{m}^{2}\right)$ & $28.6 \pm 4.8$ \\
\hline \multicolumn{2}{|l|}{ Medical history } \\
\hline Previous myocardial infarction & $233(31 \%)$ \\
\hline Previous PCI & $376(50 \%)$ \\
\hline Previous CABG & $76(10 \%)$ \\
\hline Hypertension & $645(86 \%)$ \\
\hline Hyperlipidemia & $487(65 \%)$ \\
\hline Obesity & $314(42 \%)$ \\
\hline Diabetes mellitus & $261(35 \%)$ \\
\hline $\begin{array}{l}\text { Chronic obstructive pulmonary disease/ } \\
\text { asthma }\end{array}$ & $144(19 \%)$ \\
\hline Renal impairment & $66(9 \%)$ \\
\hline \multicolumn{2}{|l|}{ Clinical findings } \\
\hline Heart rate $(\mathrm{bpm})$ & $76.8 \pm 11.8$ \\
\hline Number of angina attacks per week & $1.0 \pm 2.5$ \\
\hline Use of short-acting nitrates per week & $1.3 \pm 3.0$ \\
\hline Systolic blood pressure $(\mathrm{mmHg})$ & $137.6 \pm 15.1$ \\
\hline Diastolic blood pressure (mmHg) & $81.8 \pm 10.1$ \\
\hline \multicolumn{2}{|l|}{ Canadian Cardiovascular Society } \\
\hline Class I & $170(25 \%)$ \\
\hline Class II & $371(55 \%)$ \\
\hline Class III & $128(19 \%)$ \\
\hline Class IV & $3(<1 \%)$ \\
\hline \multicolumn{2}{|l|}{ Medication } \\
\hline Beta-blockers & $706(95 \%)$ \\
\hline Metoprolol & $664(94 \%)$ \\
\hline Ivabradine & $665(89 \%)$ \\
\hline Calcium channel blockers & $115(15 \%)$ \\
\hline Long acting nitrates & $47(6 \%)$ \\
\hline
\end{tabular}


Table 1 continued

\begin{tabular}{ll}
\hline Characteristics & $(N=747)$ \\
\hline Molsidomine & $46(6 \%)$ \\
Ranolazine & $31(4 \%)$ \\
Angiotensin-converting enzyme & $386(52 \%)$ \\
inhibitors & \\
Angiotensin II receptor antagonists & $184(25 \%)$ \\
Statins & $529(71 \%)$ \\
Aspirin & $504(68 \%)$ \\
Diuretics & $258(35 \%)$ \\
\hline
\end{tabular}

Values are means \pm standard deviations or numbers and corresponding percentages

bpm beats per minute, $P C I$ percutaneous coronary intervention, $C A B G$ coronary artery bypass graft

50/5 mg b.i.d. (47.5\%), and $25 / 5 \mathrm{mg}$ b.i.d. (35.5\%), followed by 50/7.5 mg b.i.d. (9.5\%), and $25 / 7.5 \mathrm{mg}$ b.i.d. (6.5\%). At study entry and last visit, mean daily dose of metoprolol in the FDC was 78.4 and $81.6 \mathrm{mg}$, respectively, for ivabradine it was $10.8 \mathrm{mg}$ at baseline and $11.1 \mathrm{mg}$ at the end of the study.

\section{Effectiveness}

At study entry, mean HR was $76.8 \pm 11.8 \mathrm{bpm}$ in all patients, with $\mathrm{HR} \geq 70 \mathrm{bpm}$ in $69.3 \%$ $(n=518)$. After 4 months of treatment, transition to FDC significantly reduced mean HR by $9.6 \mathrm{bpm}$ to $67.2 \pm 8.2 \mathrm{bpm}$ (Fig. 1) in the total study cohort ( $p<0.0001$ from baseline); $78.8 \%$ $(n=577)$ of all patients with available HR measurements at study end $(n=732)$ were classified as responders at the end of follow-up, defined as HR reduction to $<70 \mathrm{bpm}$ and/or absolute reduction by $\geq 10 \mathrm{bpm}$ from baseline to last visit. Total response rate (74.9\%) was very similar in magnitude for the main patient group with initial $\mathrm{HR} \geq 70 \mathrm{bpm}$.

At admission, the average number of angina attacks per week (reported 7 days prior to each visit) was $1.0 \pm 2.5$. After 4 months of therapy, the FDC of metoprolol/ivabradine led to a significant decrease of the average number of angina attacks per week to $0.2 \pm 0.9(p<0.0001$

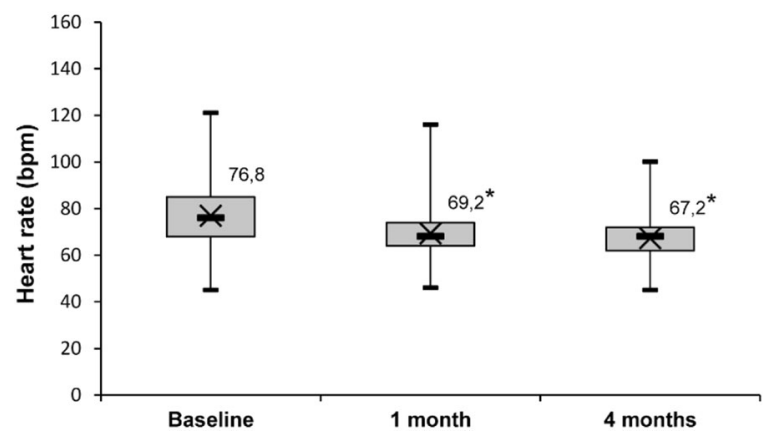

Fig. 1 Mean resting heart rate during FDC treatment over 4 months. $x$, mean; -, median; [], interquartile range; -, minimum and maximum. FDC fixed-dose combination; ${ }^{*} p<0.0001$

from baseline). Proportion of patients with $\geq 1$ angina attacks per week in the last 7 days prior to visit decreased from initially 37.8 to $6.5 \%$ after 4 months of FDC therapy (Table 2). In those 278 patients, the mean number of angina attacks was reduced from 2.7 at baseline to 0.4 after 4 months ( $p<0.0001$ from baseline), and $86.0 \%$ of them were free from limiting angina symptoms at last visit.

The average baseline consumption of short-acting nitrates per week (reported 7 days prior to each visit) was $1.3 \pm 3.0$ units. After 4 months of therapy with the FDC, average consumption of short-acting nitrates per week dropped significantly to $0.2 \pm 1.3$ units $(p<0.0001$ from baseline). Percentage of patients in need of $\geq 1$ short-acting nitrate units per week fell from $28.2 \%$ at study entry to $6.0 \%$ after 4 months (Table 2). In those 202 patients, the mean number of nitrate uptake was reduced

Table 2 Angina symptoms and short-acting nitrate use

\begin{tabular}{|c|c|c|c|c|}
\hline & \multicolumn{2}{|c|}{ Baseline } & \multicolumn{2}{|c|}{4 months } \\
\hline & $n$ & $\%$ & $n$ & $\%$ \\
\hline $\begin{aligned} \geq & 1 \text { angina } \operatorname{attack}(s) \text { per week } \\
& (n=736)\end{aligned}$ & 278 & 37.8 & 48 & $6.5^{*}$ \\
\hline $\begin{aligned} \geq & 1 \text { short-acting nitrate use }(s) \text { per } \\
& \text { week }(n=717)\end{aligned}$ & 202 & 28.2 & 43 & $6.0^{*}$ \\
\hline
\end{tabular}

Values represent patient numbers $(n)$ and corresponding percentages (\%) at baseline and study end ${ }^{*} p<0.0001$ versus baseline 
from 4.4 units at baseline to 0.7 units after 4 months ( $p<0.0001$ from baseline), and in $81.7 \%$ of them there was no further need for the use of short-acting nitrates at last visit.

At study entry, $25.3 \%$ of patients were classified as CCS grade I, $55.2 \%$ as CCS grade II, $19.1 \%$ as CCS grade III, and $0.5 \%$ as CCS grade IV. A pronounced improvement in CCS grade distribution towards lower classes was observed. At the end of follow-up, percentage of patients classified as CCS grade I increased to $63.0 \%$, while the proportion of patients in CCS class III declined to $5.4 \%(p<0.0001$ for all class changes from baseline; Fig. 2). Moreover, in the subgroup of patients with CHF or LVD at study entry, an improvement of NYHA class distribution and LVEF classification was demonstrated (data not shown).

All described effects concerning reduction of HR, number of weekly angina attacks, use of short-acting nitrates and improvement of CCS grade distribution were comparable and consistent for a number of predefined subgroups (e.g., according to gender, age $</ \geq 75$ years, heart rate, CCS grading, switch of treatment or previous PCI). The effectiveness of therapy with the metoprolol/ivabradine FDC was mostly judged as "very good" (70.6\%) or "good" (26.9\%) by the treating physicians.

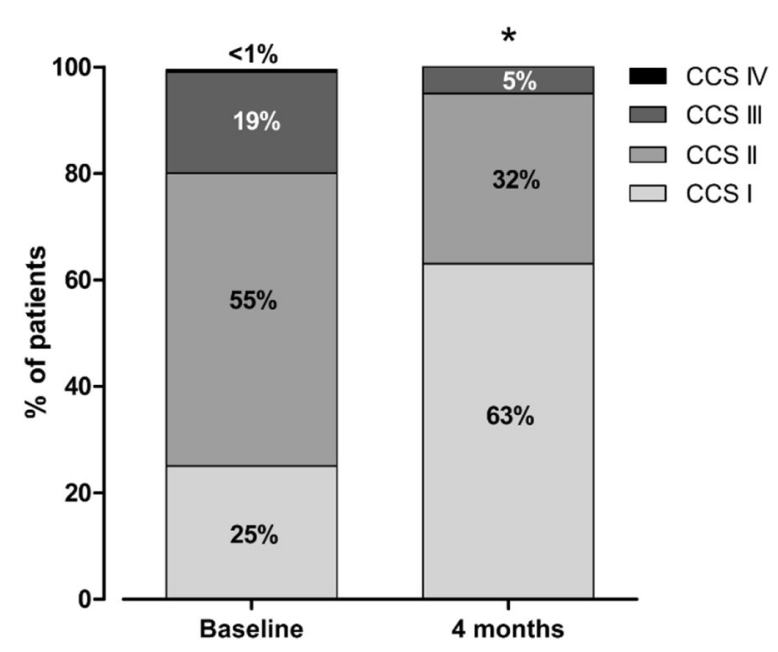

Fig. 2 Distribution of patients according to CCS class at baseline and study end. CCS Canadian Cardiovascular Society; ${ }^{*} p<0.0001$

\section{Adherence}

After 4 months of therapy with the new metoprolol/ivabradine FDC, a "complete adherence" (defined as the absence of any adherence-related problems reported in the modified fouritem Morisky questionnaire), was achieved in $58.2 \%$ of study population (Fig. 3), marking a pronounced increase compared to $33.8 \%$ of patients with this classification at study entry ( $p<0.0001$ from baseline). In particular, at the end of follow-up, $70.6 \%$ of the participants noted, that they "never" forget to take their current medication, $88.8 \%$ declared, that they never consciously decide not to take their current medication, $84.2 \%$ stated, that they never refrain from taking their current medication because of feeling good, and, finally, $71.5 \%$ declared to never have difficulties remembering when to take their current medication. With regards to all four items of the questionnaire, the remaining patients in their majority stated to be "sometimes" non-adherent, while only $\leq 1 \%$ stated to be non-adherent either "often" or "very often", respectively.

Additionally, $94.4 \%$ of patients stated that the FDC preparation is a simplification or relief in treatment of their heart disease in daily life, $88.9 \%$ noted that they are more satisfied with

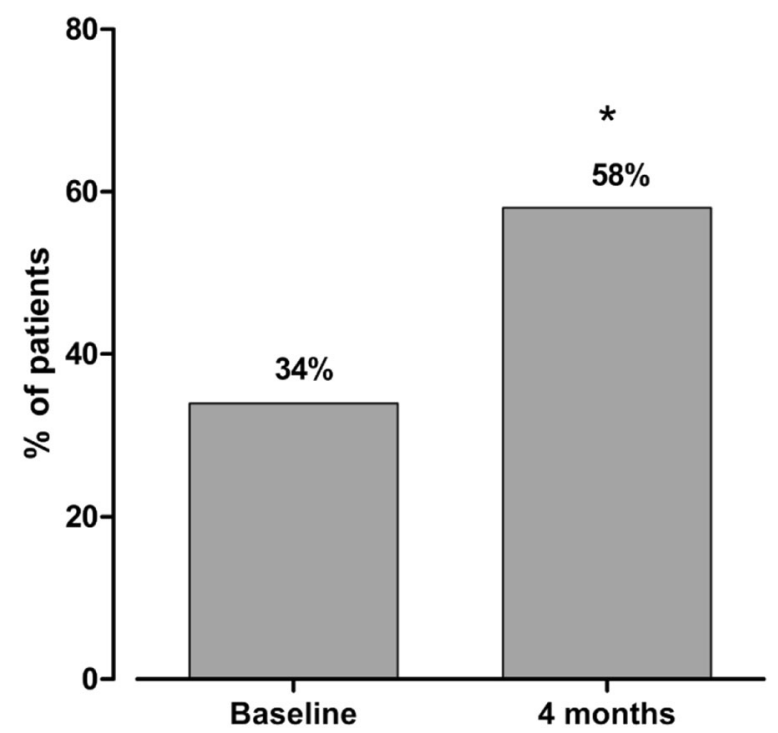

Fig. 3 Patients with "complete adherence" at baseline and study end. ${ }^{*} p<0.0001$ 
their treatment than before the switch to the FDC, and $82.4 \%$ declared that the FDC treatment improved their everyday quality of life (e.g., improved physical capacity). In line with those results, $75.2 \%$ of patients judged the tolerability of FDC therapy as "very good", and a further $23.6 \%$ as "good". Again, no marked differences in terms of medication adherence could be identified between the investigated subgroups.

\section{Safety}

Mostly mild AE occurred in $5.4 \%$ of all patients. ADR (considered related to treatment) have been reported for $2.5 \%$ of patients. No unexpected safety signals have been detected. As the most common AE dizziness, increased blood pressure, headache, and dyspnea (all $<1 \%$ ) were reported, while, e.g., bradycardia and hypotension (each $0.3 \%$ ) were just rarely seen. Serious $\mathrm{AE}$ were observed in four cases, but none of them was judged to be related to therapy. All safety aspects in the study were comparable and consistent for the above-mentioned subgroups.

\section{DISCUSSION}

In this cohort of stable AP patients already receiving an intense anti-anginal pre-treatment at study entry, switching from free combination of a beta-blocker and ivabradine to the metoprolol/ivabradine FDC formulation was associated with a relevant further reduction of heart rate, frequency of angina attacks and nitrate use, accompanied by an improvement of exercise capacity as indicated by a pronounced shift of the participants to lower CCS classes. The above-mentioned effects were consistently seen in all pre-defined subgroups, irrespective of e.g., gender, age $(</ \geq 75$ years), baseline heart rate or CCS grading, status of (pre-)treatment with ivabradine and metoprolol or history of previous PCI. Moreover, the metoprolol/ivabradine FDC was generally well tolerated by patients. Within 4 months of follow-up, a considerable improvement in self-reported medication adherence was demonstrated. Positive effects on $\mathrm{HR}$, angina symptoms and exercise tolerance (assessed by patient judgment/CCS score) are likely to be associated with the simplification of anti-anginal therapy leading to a better medication adherence in heavily pre-treated stable CAD patients with several cardiovascular and non-cardiovascular co-morbidities. The symptomatic benefits have been achieved on the basis of an already-effective concomitant anti-anginal baseline medication, indicated by the relatively low level of angina symptoms and nitrate use at study start.

In general, the beneficial effects of the metoprolol/ivabradine FDC complement those seen in the clinical ASSOCIATE (evaluation of the Antianginal efficacy and Safety of the aSsociation Of the funny Current Inhibitor ivAbradine with a beTa-blockEr) trial evaluating the free addition of ivabradine to an existing beta-blocker therapy in still symptomatic stable AP patients. After 4 months of co-therapy with ivabradine, a significant decline of HR, reduction of myocardial ischemia, and improvement of exercise capacity was demonstrated [20], supporting recent guideline recommendations for the use of such a combination in patients with uncontrolled HR $(\geq 70 \mathrm{bpm})$ and angina symptoms despite beta-blocker therapy in maximally tolerated dosage [2, 3]. Moreover, a pilot study had shown that adding ivabradine to medium-dose beta-blockers more effectively increased exercise capacity than up-titration of beta-blockers in patients with stable AP $[29,30]$. Given the known difficulties to up-titrate beta-blockers to higher doses in a real-life setting [4-7], setting a lower bar for combining them with ivabradine appears to be a viable alternative allowing lower doses of beta-blockers with less limiting side-effects.

In our study, the baseline characteristics of the population were comparable to those of three other prospective, observational cohort studies successfully testing the concept of (free) combination of beta-blockers with ivabradine in real-life populations, e.g., the ADDITIONS [21], REDUCTION (Reduction of ischemic Events by reDUCtion of heart rate In treatment Of stable aNgina with ivabradine) [22], and RESPONSIfVE (Evaluation of effectiveness and therapeutic response to ivabradine in daily 
practical use for chronic stable angina patients) study [23], with the exception of a slightly lower HR and angina frequency at study entry, which can be mainly explained by the high percentage of patients not only being pre-treated with beta-blocker but as well with ivabradine in the current study. A subgroup analysis of the ADDITIONS trial and post hoc analysis of the above-mentioned three non-interventional studies had consistently revealed a pronounced further reduction of HR, weekly angina attacks and nitrate consumption, respectively, as well as an improvement of exercise capacity and health-related quality of life (QoL) after 4 months of therapy with the free combination of metoprolol and ivabradine [24, 25]. At last follow-up, the results obtained with the new metoprolol/ivabradine FDC in terms of heart rate $(67 \mathrm{bpm})$, frequency of angina attacks per week $(0.3)$, weekly short-acting nitrate use $(0.2$ unit) and CCS class distribution were nearly identical to those with the free combination of both drugs in, e.g., the aforementioned ADDITIONS subgroup analysis [24]. Interestingly, in our study population the mean ivabradine dosage at study entry (10.8 mg) and after 4 months (11.1 mg) was roughly comparable with that used in the ADDITIONS metoprolol subgroup (9.6 and $12.6 \mathrm{mg}$, respectively), while the mean metoprolol dosage of 78.4 and $81.6 \mathrm{mg}$, respectively, was considerably lower than in the ADDITIONS cohort (102.5 and $108 \mathrm{mg}$, respectively). This finding suggests that the lower mean dosage of metoprolol used in the context of a FDC strategy in our study population may have been sufficient to achieve a similar effectiveness.

The beneficial effects of the metoprolol/ ivabradine FDC that were demonstrated after 4 months of therapy in stable AP patients mostly pre-treated with the free combination of beta-blocker and ivabradine may be mainly attributed to the observed increase in "complete adherence" to their medication from initially 34 to $58 \%$ at the end of follow-up as reported in a modified four-item Morisky questionnaire. Simplification of complex therapy regimens is a well-established concept, e.g., in cardiovascular secondary prevention or hypertension. A large meta-analysis revealed the use of FDC to significantly reduce the risk of non-adherence when compared with free combination regimens, with a decrease in relative risk of approximately 25\% [31]. Patients taking only one drug were also more likely to adhere to treatment than those on multidrug regimens, and treatment was as well more likely to be effective [32]. Another study similarly demonstrated that, after a year of taking a fixed combination of treatments, adherence was significantly higher when compared with patients taking medication separately [33]. For the metoprolol/ivabradine FDC, given twice daily, and in co-morbid CAD patients with already high tablet burden, positive effects on medication adherence can be expected to be even more pronounced. In extension to the above mentioned data our study reinforces the view that even relatively small reductions in pill burden of already heavily treated patients may generate considerable effects on adherence to therapy. In the long term, an improved adherence accompanied by an increased effectiveness could result in decreased medical costs [34].

For every single item of the modified Morisky questionnaire, absence of any self-reported adherence issues was noted for at least $70 \%$ of patients at study end. Almost $90 \%$ of patients claimed to never consciously decide not to take their medication, indicating a high satisfaction with their current therapy. Additionally, this finding is not only in line with the effectiveness of the FDC, but as well with its tolerability rated as very good/good for $98 \%$ of patients. It further confirms the physician's reporting of safety information of the FDC with only few patients experiencing AE/ADR such as phosphenes or symptomatic bradycardia. A favorable safety profile of the free combination of ivabradine and metoprolol was already observed in the aforementioned observational studies' subgroups $[24,25]$. Additionally, the medium-dose of metoprolol (maximally $2 \times 50 \mathrm{mg} /$ day) used in the FDC may as well have contributed to the low rate of side-effects. Although not directly addressed in our study, patient answers on satisfaction with FDC therapy and its influence on their therapy management, reduction of symptoms and everyday quality of life indicate further positive effects on health-related QoL. 


\section{Limitations}

One limitation of this study was its relatively short duration of 4 months. Although the follow-up period seems to be fully sufficient for assessment of effectiveness and tolerability of the FDC [21-23], data on evaluation of medication adherence in such a setting are not available. As well, assessment of medication adherence was performed indirectly by analyzing a modified, four-item Morisky patient questionnaire. Possible effects on health-related QoL were not evaluated with validated measurement scales. To a minor degree, in patients taking only one of the two drugs at study entry, additional effects of switching to the FDC cannot be ruled out, although subgroup analyses did not reveal any relevant influence of baseline medication on the overall results. Additionally, this was an open-label study without a placebo or free metoprolol/ivabradine combination for comparison of treatment effects of the metoprolol/ivabradine FDC.

The main strength of this study was that it was carried out in a large outpatient population of stable AP patients in 260 centers in Germany. Furthermore, due to the non-interventional design of the study, it was possible to assess treatment in routine clinical practice in terms of effectiveness, safety and medication adherence. Patient groups usually underrepresented in controlled clinical trials (e.g., elderly, women, patients with multiple co-morbidities) can only be covered and evaluated by such open-label, observational cohort studies [35].

\section{CONCLUSIONS}

In this cohort of stable AP patients in a real-life setting, presenting with already intense anti-anginal pre-treatment at baseline, therapy with a metoprolol/ivabradine FDC preparation was associated with a further reduction in HR by $10 \mathrm{bpm}$. At study end, a clinically relevant reduction of angina symptoms and nitrate consumption by more than $80 \%$ was observed. Moreover, exercise capacity was markedly improved as reflected by a significant shift of patients towards lower CCS classes. The tolerability of the FDC was favorable with no unexpected safety signals during follow-up.

Results were consistently confirmed in different subgroups, including patients $\geq 75$ years of age, with high CCS grading or previous PCI. These positive effects may be mainly mediated by the pronounced improvement in medication adherence associated with use of the FDC. Taken together, in stable AP patients pre-treated with beta-blocker (e.g., metoprolol) and ivabradine the metoprolol/ivabradine FDC improves medication adherence and increases symptom control in clinical practice.

\section{ACKNOWLEDGEMENTS}

Sponsorship and article processing charges for this study were funded by Servier Deutschland $\mathrm{GmbH}$, Munich, Germany. All authors had full access to all of the data in this study and take complete responsibility for the integrity of the data and accuracy of the data analysis. All named authors meet the ICMJE criteria for authorship for this manuscript, take responsibility for the integrity of the work as a whole, and have given final approval for the version to be published. The authors would like to thank all investigators for their contributions to the study, and Dr Michael Lohmann for assistance in the preparation of the manuscript. Subsets of these data have been presented as posters at the Annual Congress of the German Cardiac Society in 2017 and the Annual Congress of the German Society of Internal Medicine in 2017.

Disclosures. Dr. Dimitar Divchev received honoraria for lectures and as scientific coordinator of this study from Servier. Dr. Georg Stöckl is an employee of Servier Deutschland $\mathrm{GmbH}$, Munich (Medical Affairs Department).

Compliance with Ethics Guidelines. All procedures followed were in accordance with the ethical standards of the responsible committee on human experimentation (institutional and national) and with the Helsinki Declaration of 1964, as revised in 2013. Informed consent was obtained from all 
patients for being included in the study. Ethical approval was granted by the independent ethics commission in Freiburg/Germany (FEKI).

Data availability. The datasets generated during and/or analyzed during the current study are available from the corresponding author on reasonable request.

Open Access. This article is distributed under the terms of the Creative Commons Attribution-NonCommercial 4.0 International License (http://creativecommons.org/licenses/ by-nc/4.0/), which permits any noncommercial use, distribution, and reproduction in any medium, provided you give appropriate credit to the original author(s) and the source, provide a link to the Creative Commons license, and indicate if changes were made.

\section{REFERENCES}

1. Heusch G. Heart rate in the pathophysiology of coronary blood flow and myocardial ischemia: benefit from selective bradycardic agents. $\mathrm{Br} \mathrm{J}$ Pharmacol. 2008;153:1589-601.

2. Montalescot G, Sechtem U, Achenbach S, Andreotti F, Arden C, et al. 2013 ESC guidelines on the management of stable coronary artery disease: the Task Force on the management of stable coronary artery disease of the European Society of Cardiology. Eur Heart J. 2013;34:2949-3003.

3. Fihn SD, Gardin JM, Abrams J, Berra K, Blankenship JC, et al. 2012 ACCF/AHA/ACP/AATS/PCNA/SCAI/ STS guideline for the diagnosis and management of patients with stable ischemic heart disease: a report of the American College of Cardiology Foundation/ American Heart Association Task Force on practice guidelines, and the American College of Physicians, American Association for Thoracic Surgery, Preventive Cardiovascular Nurses Association, Society for Cardiovascular Angiography and Interventions, and Society of Thoracic Surgeons. Circulation. 2012;126:e354-471.

4. Daly CA, Clemens F, Sendon JL, Tavazzi L, Boersma E, Danchin N, Delahaye F, Gitt A, Julian D, Mulcahy D, Ruzyllo W, Thygesen K, Verheugt F, Fox KM. Inadequate control of heart rate in patients with stable angina: results from the European heart survey. Postgrad Med J. 2010;86:212-7.
5. Wiest FC, Bryson CL, Burman M, McDonell MB, Henikoff JG, Fihn SD. Suboptimal pharmacotherapeutic management of chronic stable angina in the primary care setting. Am J Med. 2004;117:234-41.

6. Tendera M, Fox K, Ferrari R, Ford I, Greenlaw N, Abergel H, Macarie C, Tardif JC, Vardas P, Zamorano J, Gabriel SP. Inadequate heart rate control despite widespread use of beta-blockers in outpatients with stable CAD: findings from the international prospective CLARIFY registry. Int J Cardiol. 2014;176:119-24.

7. Steg PG, Ferrari R, Ford I, et al. Heart rate and use of beta blockers in stable outpatients with coronary artery disease. PLoS One. 2012;7(5):e36284.

8. DiFrancesco D. Cardiac pacemaker If current and its inhibition by heart rate-reducing agents. Curr Med Res Opin. 2005;21:1115-22.

9. DiFrancesco D, Camm AJ. Heart rate lowering by specific and selective If current inhibition with ivabradine. A new therapeutic perspective in cardiovascular disease. Drugs. 2004;64:1757-65.

10. Thollon C, Vilaine JP. $I$ f inhibition in cardiovascular diseases. Adv Pharmacol. 2010;59:53-92.

11. Camici PG, Gloekler S, Levy BI, Skalidis E, Tagliamonte E, Vardas P, Heusch G. Ivabradine in chronic stable angina: effects by and beyond heart rate reduction. Int J Cardiol. 2016;215:1-6.

12. Dillinger JG, Maher V, Vitale C, Henry P, Logeart D, Manzo SS, Allee G, Levy BI. Impact of ivabradine on central aortic blood pressure and myocardial perfusion in patients with stable coronary artery disease. Hypertension. 2015;66:1138-44.

13. Tagliamonte E, Cirillo T, Rigo F, Astarita C, Coppola A, Romano C, Capuano N. Ivabradine and bisoprolol on Doppler-derived coronary flow velocity reserve in patients with stable coronary artery disease: beyond the heart rate. Adv Ther. 2015;32:757-67.

14. Mangiacapra F, Colaiori E, Ricottini E, Balducci F, Creta A, Demartini G, Di Sciascio G. Heart rate reduction by ivabradine for improvement of endothelial function in patients with coronary artery disease: the RIVENDEL study. Clin Res Cardiol. 2017;106(1):69-75.

15. Gloekler S, Traupe T, Stoller M, Schild D, Steck H, Khattab A, Vogel R, Seiler C. The effect of heart rate reduction by ivabradine on collateral function in patients with chronic stable coronary artery disease. Heart. 2014;100:160-6.

16. Borer JS, Fox K, Jaillon P, Lerebours G. Antianginal and antiischemic effects of ivabradine, an $I \mathrm{f}$ 
inhibitor, in stable angina: a randomized, double-blind, multicentered, placebo-controlled trial. Circulation. 2003;107:817-23.

17. Lopez-Bescos L, Filipova S, Martos R. Long term safety and efficacy of ivabradine in patients with chronic stable angina. Cardiology. 2007;108:387-96.

18. Ruzyllo W, Tendera M, Ford I, Fox KM. Antianginal efficacy and safety of ivabradine compared with amlodipine in patients with stable effort angina pectoris: a 3-month randomised, double-blind, multicentre, noninferiority trial. Drugs. 2007;67:393-405.

19. Tardif JC, Ford I, Tendera M, Bourassa MG, Fox K. Efficacy of ivabradine, a new selective $I$ f inhibitor, compared with atenolol in patients with chronic stable angina. Eur Heart J. 2005;26:2529-36.

20. Tardif JC, Ponikowski P, Kahan T. Efficacy of the If current inhibitor ivabradine in patients with chronic stable angina receiving beta blocker therapy: a 4 month, randomized, placebo-controlled trial. Eur Heart J. 2009;30:540-8.

21. Werdan K, Ebelt H, Nuding S, Höpfner F, Hack G, Müller-Werdan U. Ivabradine in combination with beta-blocker improves symptoms and quality of life in patients with stable angina pectoris: results from the ADDITIONS study. Clin Res Cardiol. 2012;101:365-73.

22. Köster R, Kaehler J, Meinertz T. Treatment of stable angina pectoris by ivabradine in everyday practice: the REDUCTION study. Am Heart J. 2009; 158:e51-7.

23. Perings S, Stöckl G, Kelm M. Effectiveness and tolerability of ivabradine with or without concomitant beta-blocker therapy in patients with chronic stable angina in routine clinical practice. Adv Ther. 2016;33:1550-64.

24. Werdan K, Ebelt H, Nuding S, Höpfner F, Stöckl G, Müller-Werdan U. Ivabradine in combination with metoprolol improves symptoms and quality of life in patients with stable angina pectoris: a post hoc analysis from the ADDITIONS trial. Cardiology. 2016;133:83-90.

25. Werdan K, Perings S, Köster R, Kelm M, Meinertz T, Stöckl G, Müller-Werdan U. Effectiveness of ivabradine treatment in different subpopulations with stable angina in clinical practice: a pooled analysis of observational studies. Cardiology. 2016;135:141-50.
26. Kulkarni SP, Alexander KP, Lytle B, Heiss G, Peterson ED. Long-term adherence with cardiovascular drug regimen. Am Heart J. 2006;151:185-91.

27. Choudhry NK, Fischer MA, Avorn J, Liberman JN, Schneeweiss S, Pakes J, Brennan TA, Shrank WH. The implications of therapeutic complexity on adherence to cardiovascular medications. Arch Intern Med. 2011;171:814-22.

28. Morisky DE, Green LW, Levine DM. Concurrent and predictive validity of a self-reported measure of medication adherence. Med Care. 1986;24:67-74.

29. Amosova E, Andrejev E, Zaderey I, Rudenko U, Ceconi C, Ferrari R. Efficacy of ivabradine in combination with beta-blocker versus uptitration of beta-blocker in patients with stable angina. Cardiovasc Drugs Ther. 2011;25:531-7.

30. Sy RW, Freedman SB. Beta-blocker dose uptitration or addition of ivabradine in stable angina: more is not necessarily better. Editorial to: 'Efficacy of ivabradine in combination with beta-blocker versus uptitration of betablocker in patients with stable angina' by E. Amosova et al. Cardiovasc Drugs Ther. 2011;25:501-2.

31. Bangalore S, Kamalakkannan G, Parkar S, Messerli FH. Fixed-dose combinations improve medication compliance: a meta-analysis. Am J Med. 2007;120:713-9.

32. Fung V, Huang J, Brand R, Newhouse JP, Hsu J. Hypertension treatment in a Medicare population: adherence and systolic blood pressure control. Clin Ther. 2007;29:972-84.

33. Patel BV, Leslie RS, Thiebaud P, Nichol MB, Tang SS, Solomon H, Honda D, Foody JM. Adherence with single-pill amlodipine/atorvastatin vs. a two-pill regimen. Vasc Health Risk Manag. 2008;4:673-81.

34. Muszbek N, Brixner D, Benedict A, Keskinaslan A, Khan ZM. The economic consequences of noncompliance in cardiovascular disease and related conditions: a literature review. Int J Clin Pract. 2008;62:338-51.

35. Vitale C, Fini M, Spoletini I, Lainscak M, Seferovic $\mathrm{P}$, Rosano GMC. Under-representation of elderly and women in clinical trials. Int $\mathrm{J}$ Cardiol. 2017;232:216-21. 